\title{
EDITORIAL
}

\section{Artificial intelligence in intensive care medicine}

\author{
Muhammad Mamdani $i^{1,2,3^{*}}$ and Arthur S. Slutsky ${ }^{4,5^{*}}$ [C]
}

(C) 2020 Springer-Verlag GmbH Germany, part of Springer Nature

Advances in technology, data, and analytics are transforming society. Amazon has transformed the way we shop. Uber has upended the taxi industry. The largest hotel chain in the world is Airbnb. Healthcare, which is among the most data-rich sectors, has been slow to adapt. Further, emerging trends in virtual healthcare and artificial intelligence (AI) will soon challenge how we function as clinicians and healthcare systems. For example, machine learning algorithms have been able to classify echocardiograms as accurately as board-certified echocardiographers [1] and perform at least as well as experienced clinicians in predicting the need for early revascularization in patients with suspected coronary artery disease [2]. Indeed, AI-enabled clinical tools are now rapidly entering the commercial market [3]. An Intel survey of approximately 200 healthcare decision-makers in the United States indicated that more than half of respondents expect widespread AI adoption by 2023 and more than one-third are already using AI-based solutions in their settings [4].

Briefly, AI has been defined as the theory and development of computer systems able to perform tasks normally requiring human intelligence, such as visual perception, speech recognition, decision-making, and translation between languages [5]. Machine learning methods have formed the foundation of modern AI, although fundamental machine learning principles such as neural networks have existed since the 1940s [6]. Recent advances

*Correspondence: Muhammad.mamdani@unityhealth.to; Arthur. slutsky@unityhealth.to

${ }^{1}$ Unity Health Toronto, Li Ka Shing Knowledge Institute, St. Michael's Hospital, 30 Bond Street, Toronto, ON M5B 1W8, Canada

${ }^{4}$ Keenan Research Center for Biological Science, Li Ka Shing Knowledge Institute, St. Michael's Hospital, Unity Health Toronto, 30 Bond Street, Toronto, ON M5B 1W8, Canada

Full author information is available at the end of the article in data digitization, storage, and processing, however, have enabled these powerful methods to fuel modern AI initiatives.

Intensive care medicine is particularly well positioned to harness these advances given the abundance of clinical, physiological, and laboratory data constantly generated in the intensive care unit (ICU) [7]. However, the intensive care environment is complicated by rapidly changing patient status and care, which poses special challenges for the development and deployment of AI. For example, 'real time' data may be needed to drive the AI algorithms and learning approaches would need to adapt to rapid changes in clinical management. The nuances of 'real-world practice' have limited the application of AI solutions that are often developed using ideal data in ideal environments that result in insufficient 'real world performance' and limited clinical relevance $[8,9]$. Further, the clinician understanding of AI is limited. Several surveys have suggested that the majority of medical students and staff clinicians view themselves as novices in the understanding of AI $[10,11]$. To address the need for better clinician understanding of AI, we have created a series of articles focused on three themes in applied AI in medicine, namely enabling data, AI development, and AI deployment (Fig. 1).

Enabling data: AI methods often require very large amounts data, such as those routinely generated from a variety of data sources including monitors that track physiologic data, frequent assessment of vitals, laboratory values, and medications. Ideally, these data are available digitally (as opposed to paper), of high quality, and easily linked to a given patient. The reality, however, is that key data are often missing, or are of poor quality, resulting in the development of sub-optimal AI models. The process of 'cleaning' and 'structuring' data to make them 'AI-ready' can be timeconsuming and tedious. Ultimately, applied AI models

\section{Springer}




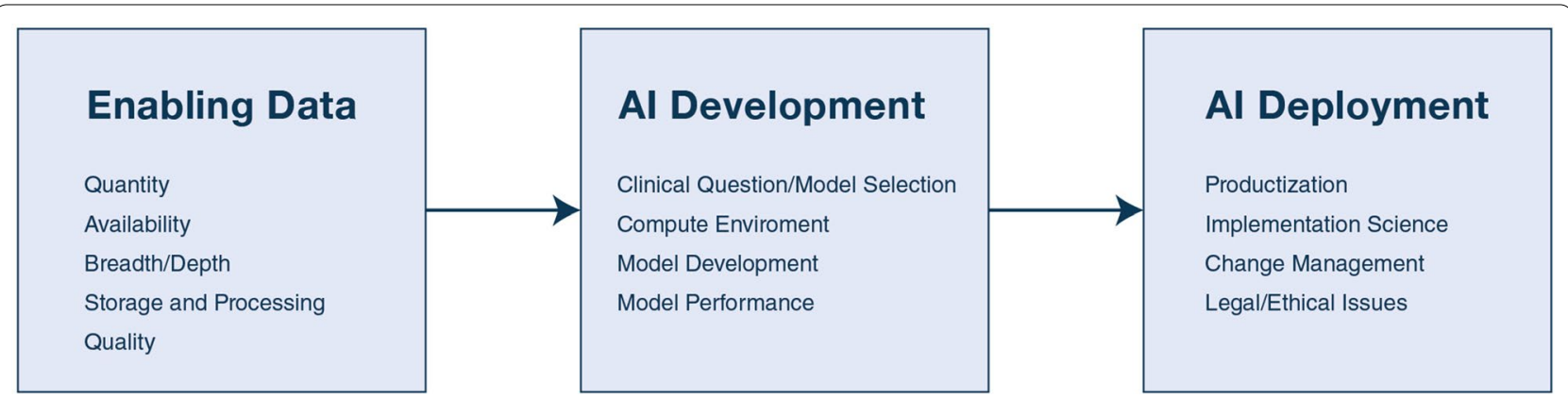

Fig. 1 Development and deployment of Al tools into clinical practice

will, to a certain extent, need to embrace the imperfections of real-world data rather than focusing on their ideal state to be useful. Further, many hospitals do not have suitable data environments where very large volumes of data can be safely captured, stored, and processed rapidly for purposes of AI development. Data and infrastructure requirements to enable AI development in the ICU will be a key focus in our series on AI in intensive medicine.

AI development: Although the development of $\mathrm{AI}$ models is predicated on the availability of high quality, reliable data, of equal or greater importance is clearly articulating a relevant clinical or operational question that can be addressed by AI methods with the available data. The identified question, which may be limited by the quality and availability of data, as well as a sufficient compute environment, will then drive AI model selection. For example, reinforcement learning approaches may be best suited for determining optimal dosing strategies for medications such as sedatives whose dosing parameters change frequently. Such machine learning approaches can be quite intensive computationally and require significant processing power that many hospitals do not routinely possess. Further, understanding the performance of AI models to determine clinical utility can be challenging. Model performance parameters such as precision, recall, F1 score, and area under the precision-recall curve (AUPRC) in classification models and $R^{2}$ and root mean squared error (RMSE) in linear regression models can assist clinicians in understanding how clinically useful specific models may be. When developing AI models, critical issues such as social justice and bias also need to be considered. AI models are routinely trained on data reflecting biases that are inherent in modern society. For example, an algorithm used on approximately 200 million Americans to identify patients for 'high-risk care management programs' has been shown to considerably disadvantage Black patients [12]. Such important concepts on AI model development will also be reviewed in our series on AI in intensive medicine.

AI deployment: The most compelling AI models addressing highly important clinical issues may be irrelevant clinically if a proper implementation plan is not developed and executed. Implementation of AI solutions can be complex. Understanding human factors principles that define how humans interact with AI tools in a positive manner, design and workflow considerations that foster engagement, and change management are all part of implementation science that drive AI solution adoption. In addition, legal and ethical considerations relevant to AI solutions are constantly evolving. For example, the issue of legal liability in a situation where an AI solution accurately predicts an adverse patient outcome that was not acted upon can be challenging but needs to be addressed prior to AI solution deployment. Finally, the evaluation of AI deployment is critical to ensure the intended benefits are realized. An evaluation of a clinical prediction rule used to guide management of febrile urinary tract infections found a greater than four-fold increase in secondary hospitalizations following initial outpatient treatment [13].

While the development and deployment of AI solutions in intensive care medicine may seem daunting-and as highlighted in a recent debate in this Journal-somewhat controversial $[14,15]$, they offer considerable potential to improve the quality of care we provide to our patients. We hope this series will not only inform intensivists on the development and application of AI but will enable our community to be informed consumers of this potentially transformative technology. And of course, most importantly, the major goal of this series is to improve patient outcomes while optimizing the use of healthcare resources. With these issues in mind, we would like to invite contributions from the AI and intensive medicine 
communities for potential publication in association with this series.

\begin{abstract}
Author details
${ }^{1}$ Unity Health Toronto, Li Ka Shing Knowledge Institute, St. Michael's Hospital, 30 Bond Street, Toronto, ON M5B 1W8, Canada. ${ }^{2}$ Faculty of Medicine, University of Toronto, Toronto, Canada. ${ }^{3}$ Leslie Dan Faculty of Pharmacy, University of Toronto, Toronto, Canada. ${ }^{4}$ Keenan Research Center for Biological Science, Li Ka Shing Knowledge Institute, St. Michael's Hospital, Unity Health Toronto, 30 Bond Street, Toronto, ON M5B 1W8, Canada. ${ }^{5}$ Interdepartmental Division of Critical Care Medicine, University of Toronto, Toronto, Canada.
\end{abstract}

\section{Compliance with ethical standards}

\section{Conflicts of interest}

The authors declare that they have no conflict of interest in relation to this paper.

\section{Publisher's Note}

Springer Nature remains neutral with regard to jurisdictional claims in published maps and institutional affiliations.

Received: 30 June 2020 Accepted: 24 July 2020

Published online: 7 August 2020

\section{References}

1. Madani A, Arnaout R, Mofrad M, Arnaout R (2018) Fast and accurate view classification of echocardiograms using deep learning. NPJ Digit Med 1:6

2. Arsanjani R, Dey D, Khachatryan T et al (2015) Prediction of revascularization after myocardial perfusion SPECT by machine learning in a large population. J Nucl Cardiol. 22(5):877-884
3. https://www.docwirenews.com/docwire-pick/future-of-medicine-picks/ fda-approved-uses-of-ai-in-healthcare/. Accessed 1 Dec 2019

4. https://newsroom.intel.com/wp-content/uploads/sites/11/2018/07/healt hcare-iot-infographic.pdf. Accessed 1 Dec 2019

5. https://www.lexico.com/en/definition/artificial_intelligence

6. https://cs.stanford.edu/people/eroberts/courses/soco/projects/neura I-networks/History/history1.html

7. Komorowski M (2019) Artificial intelligence in intensive care: are we there yet? Intensive Care Med 45:1298-1300

8. Michard F, Teboul JL (2019) Predictive analytics: beyond the buzz. Ann Intensive Care 9:46

9. Desai AN (2020) Artificial intelligence: promise, pitfalls, and perspective. JAMA 323:2448-2449

10. Ooi SKG, Makmur A, Soon AYQ et al (2019) Attitudes toward artificial intelligence in radiology with learner needs assessment within radiology residency programmes: a national multi-programme survey. Singapore Med J. https://doi.org/10.11622/smedj.2019141

11. Pinto Dos Santos D, Giese D, Brodehl S et al (2019) Medical students' attitude towards artificial intelligence: a multicentre survey. Eur Radiol. 29(4):1640-1646

12. Obermeyer Z, Powers B, Vogeli C, Mullainathan S (2019) Dissecting racial bias in an algorithm used to manage the health of populations. Science 366(6464):447-453

13. Stalenhoef JE, van der Starre WE, Vollaard AM, Steyerberg EW et al (2017) Hospitalization for community-acquired febrile urinary tract infection: validation and impact assessment of a clinical prediction rule. BMC Infect Dis 17(1):400

14. Komorowski M (2020) Clinical management of sepsis can be improved by artificial intelligence: yes. Intensive Care Med 46:375-377

15. Garnacho-Montero J, Martin-Loeches I (2020) Clinical management of sepsis can be improved by artificial intelligence: no. Intensive Care Med $46: 378-380$ 